\title{
A Thermal Analysis of the Horizontal Zone Refining of Indium Antimonide
}

G. S. Roussopoulos, P. A. Rubini*

School of Engineering, Cranfield University,

Cranfield, Bedfordshire, MK43 0AL, UK

Tel. +44 (0) 1234754649

Email: p.a.rubini@cranfield.ac.uk

* Corresponding author

Author Keywords: A1. Computer simulation; A1. Heat transfer; A1. Conduction; A1. Convection A2. Zone melting and refining;

PACS classification codes: 81.10.Fq; 81.05.Ea; 44.10.+I; 44.30.+v 


\begin{abstract}
Single crystals of semiconductors and optical materials are very important in the field of computer technology and communications. The properties of such crystalline materials significantly depend upon the quantity and the type of impurities contained in their structure. The principal objective of this work was to further the understanding of the heat transfer mechanisms in the horizontal zone refining process and, by successfully modelling the process, to obtain a correlation between the length of the molten zone and typical operational parameters, for example, the physical dimensions of the process equipment, the heating element and the required energy input. Results are presented from the associated parametric study accomplished through a three-dimensional pseudosteady state simulation of the zone refining process for Indium Antimonide (InSb). The conclusions will be of value when optimising the operating efficiency of horizontal zone refining where the length of the molten zone is a significant factor.
\end{abstract}




\section{Introduction}

The general term zone refining denotes a family of methods for controlling the distribution of soluble impurities or solutes in crystalline materials. In such methods a short molten zone travels slowly through a relatively long solid charge and, while traveling, redistributes the solutes in the charge [1]. This is a very important process, since the properties of such crystalline materials depend heavily on the quantity and the type of impurities contained in their structure. In multipass zone refining, a long solid ingot is set in relative motion with a number of closely placed heating elements, providing the energy input necessary for the redistribution of solutes in the ingot to take place. The advantage of this variation of the zone refining process is mainly the savings in the operating time, as succeeding crystallization begins before the preceding one is completed [1].

A number of researchers have investigated methods of improving the efficiency of zone refining by optimising parameters such as the length of the molten zone, or the number of passes [2-5]. Chang et al. [6] focused their research in the coupling of the buoyancy driven heat transfer within a vertical molten zone and the positions of the interfaces relative to the heaters, in poor conductors. Kobayashi [7] also studied the relation between the power required to produce a molten zone and the zone shape. Hoe Wang et al. [8] developed a two-dimensional pseudo-steady-state model for zone melting recrystallization in which conduction in solid and molten silicon and $\mathrm{SiO}_{2}$, convection in molten silicon and radiation are included. Louchev [9] examined the influence of natural convection on the formation of a molten zone under optical heating.

The objective of this work was to develop a complete model for the heat transfer processes in the multi-pass horizontal zone refining of a semiconducting material, using 
a commercial computational fluid dynamics package, FLUENT ver.6.1 [10]. Considering that the length of the molten zone is one of the most important factors when optimising the purification method, reaching a correlation relating the energy input to the material and the length of the molten zone created, for different specified dimensions of the equipment, is expected to be of significant importance for the efficient operation and optimisation of the process equipment. Also, the influence of the inclination of the equipment, on the heat transfer aspect of the process, was examined.

\section{Description of the equipment}

The multi-pass zone refining process equipment studied is illustrated in Figures 1 and 2. The semiconducting material, in this case Indium Antimonide (InSb), is placed inside a closed Quartz $\left(\mathrm{SiO}_{2}\right)$ tube. Six heating elements are equally distributed along the length of the tube with a spacing of approximately $150 \mathrm{~mm}$. The tube is $1480 \mathrm{~mm}$ long with a diameter of $50 \mathrm{~mm}$ in the base configuration. In the specified equipment the moving part of the process is the tube. Each heating element is $50 \mathrm{~mm}$ wide constructed from a spiral coil within a ceramic block. The tube is inclined at approximately 6 degrees to increase the efficiency of the purification process. For anticorrosion purposes the remaining volume of the tube is filled with Hydrogen. The level of the semiconducting material enclosed is approximately $15 \mathrm{~mm}$. 


\section{Methodology}

Simulations were accomplished in three stages of increasing complexity. In the preliminary stage, a simplified three-dimensional, pseudo-steady state case, modelling only the heat transfer within the semiconducting material was carried out, i.e. conduction only. During this stage radiative transfer from the free surface of the charge, natural convection within the atmosphere of quartz tube and the inclination of the tube, were ignored. Similar simplified models have been used in previous investigations when investigating the influence of the length of the molten zone on the process efficiency. This model provides a basis against which a more detailed model may be compared.

The furnace was assumed to be comprised of six identical heating zones, see Figure 3, thereby implying that the ends of the furnace did not significantly effect the heat transfer distribution, and that the energy input from each one of the six equally spaced heaters was identical. Consequently only a single heating zone, $150 \mathrm{~mm}$ in length, was modelled, with prescribed adiabatic boundary conditions at either end, see Figure 3. Although natural convection was not simulated at this stage, to be consistent with the later simulations, FLUENT V6 [10] was also employed for the conduction calculations. A hexahedral mesh with approximately 34000 elements was employed to represent only the semiconductor charge. A non-uniform mesh was utilised to resolve the region neighbouring the heater elements in more detail.

In the second stage, laminar natural convection within the hydrogen atmosphere and radiative heat transfer from the heater elements and through the quartz tube were taken into account in the three-dimensional model. As before only a single heater 
element was modelled with symmetry boundary conditions at either end, and the inclination of the tube was ignored.

Radiative heat transfer from the heater element was simulated using a surfaceto-surface model with pre-computed view factors. The model assumed a nonparticipating medium, i.e. that absorption, emission and scattering could be neglected, and that the surfaces could be assumed to be optically grey and diffuse [10].

Computational grids ranging from 100000 to 180000 hexahedral cells were employed to represent both the semiconductor charge and the hydrogen atmosphere, according to the diameter of the tube. Second order accurate interpolation was employed for the convective terms in all simulations with the final mesh densities being selected after preliminary assessment of the independence of the results from the computational grid. Steady state simulations were carried out using the semi-implicit segregated pressure correction solver, assuming that the density of the hydrogen varied only as a function of temperature, [10]. The default convergence criteria within Fluent were disabled and simulations continued until no significant change in the temperature fields could be determined between iterations. Typically, solutions were obtained in a few hours on a $2.4 \mathrm{GHz}$ Pentium 4 PC running Linux.

Since the rate of motion of the tube during the zone refining process is relatively slow, all the simulations were considered to be pseudo-steady state. As a consequence the change of phase, and hence latent heat effects, and the progression of the molten region was not taken into account. Furthermore the natural convection inside the molten zone was assumed to be relatively insignificant in comparison to the incident radiative heat flux and only internal conduction within the semiconductor charge was taken into account. 
The thermal properties of InSb are detailed in Table 1. In the simulations the phase of the mixture was not explicitly tracked, rather the phase interface was defined by an isotherm at the melting point, as predicted by the simulation, and physical properties expressed as a function of temperature.

The thermal conductivity of the liquid and solid phases of InSb is significantly different. Therefore, for temperatures below $750 \mathrm{~K}$, i.e. pure solid, the value of the solid InSb was employed, for temperatures above $875 \mathrm{~K}$, i.e. pure liquid, the value of the liquid InSb was used, between those limits linear interpolation was employed. A less pronounced difference in the specific heat capacity exists between the liquid and solid phases, a constant value equal to that of the solid phase was assumed to apply everywhere.

A parametric investigation was carried out to investigate the effect of the width of the heating element and the diameter of the quartz tube on the length of the molten zone. Three tube diameters of $50 \mathrm{~mm}, 75 \mathrm{~mm}$ and $100 \mathrm{~mm}$ were considered with heater widths from $50 \mathrm{~mm}$ to $100 \mathrm{~mm}$ in $10 \mathrm{~mm}$ increments. In each case the relative proportion of the semiconducting material was kept constant.

In the final stage, the whole tube, comprising six heaters and a six degree inclination was modelled to quantify the previous assumption of modelling only a single heater zone.

\section{Boundary conditions}

A uniform heat flux was chosen to represent the radiative heat flux from the heating element on the surface of the quartz tube immediately adjacent to the heater element itself, with a width equal to that of the heating element. Given the relatively 
close spacing between the heater element and the tube, see Figure 2, this was seen to be an acceptable approximation. The heat flux was varied as part of the parametric study, the results from which are presented in the following section.

The upper and lower surfaces of the quartz tube were assumed to receive identical incident heat fluxes from the enclosing heater element. The far ends of the zone were treated as adiabatic boundaries under the assumption that they were bounded by an identically heated region.

The remaining boundaries were modelled with a mixed convective and radiative boundary condition. A convective heat transfer coefficient equal to $9.5 \mathrm{~W} / \mathrm{m}^{2} \mathrm{~K}$ was specified over the exterior surfaces, based upon an approximate value for natural convection to a horizontal cylinder. The quartz tube was assumed to have an emissivity of 0.5 and the Indium Antimonide an emissivity of 0.55 .

During the preliminary stage of the simulations the atmosphere above the semiconductor charge was assumed to be at a constant temperature of $600 \mathrm{~K}, 200 \mathrm{~K}$ less than the melting point of the material. The temperature of the exterior ambient air below the bottom-curved surfaces was assumed to be $300 \mathrm{~K}$. The quartz walls of the tube were assumed to have a uniform thickness of $2 \mathrm{~mm}$. In the more detailed stages the temperature of the hydrogen atmosphere was predicted and the external ambient boundary conditions were applied over the whole of the quartz tube. Full details of the simulation procedure and the results obtained may be found in Roussopolous [11].

\section{Results and discussion}

The temperature distribution on the vertical mid-plane, at mid-height within the semiconducting material, was selected for comparative purposes. The length of the 
molten zone within the Indium Antimonide was estimated from the isotherm at the melting point. Validation of the predicted molten zone length was achieved by comparison with qualitative measurements obtained by visual inspection of the operating equipment [12].

A parametric study was carried out, with simulations for varying geometrical dimensions of the quartz tube, width of the heaters and energy inputs. The results from the study allowed a correlation to be obtained between the selected parameters and the length of the molten zone in the semiconductor charge.

Figure 4 presents the temperature distribution for the $50 \mathrm{~mm}$ tube and a heating element of $50 \mathrm{~mm}$ width, on the mid-plane, at mid-height in the semiconductor charge. These results include the effects of natural convection in the atmosphere and radiative heat transfer from the heater elements. The length of the zone containing the molten material was estimated from the isotherm at the melting point. As shown in the figure, by successively increasing the output of the heater element, a heat input of approximately $0.094 \mathrm{~kW}$ per heater is needed for the Indium Antimonide to attain its melting point. For convenience the heat input is also presented in a normalized form with respect to the minimum energy input required to reach the melting point.

The predicted temperature distribution on the surface of the quartz tube is illustrated in Figure 5a and on a vertical plane mid-way through the tube in Figure 5b. From the isotherms, the shape of the molten zone in the centre of the semiconducting material can be readily identified. The predicted shape is consistent with that expected when the heater elements are in close proximity to the quartz tube.

Figure 6 illustrates the predicted velocity distribution within the hydrogen atmosphere on the same vertical plane, mid-way through the tube, with two 
recirculating cells formed under the heating element. Hydrogen is convected away from the upper heated surface of the quartz tube to the colder surface of the semiconducting material. The results are in qualitative agreement with those obtained from previous work on the simulation of natural convection in a rectangular air filled enclosure [11].

The sensitivity of the results to the assumed boundary conditions was checked by varying both the external heat transfer coefficient and the surface emissivities. A $20 \%$ variation in the assumed values resulted in approximately a $20 \mathrm{~K}$ variation in the temperature of the semiconductor charge. As a result any errors in the assumptions were assumed to have only a second order effect on the results.

The results from the preliminary investigation, where natural convection and radiative heat transfer were neglected, are not shown. These were found to significantly under predict the required heat input (for example only $5 \mathrm{~kW} / \mathrm{m}^{2}$ being required rather than $12 \mathrm{~kW} / \mathrm{m}^{2}$ to melt the charge), due to not accounting for radiative loss from the semiconductor charge. Whilst not surprising the conclusion is important whilst selecting thermal boundary conditions when modelling the movement of the melt zone interface.

The results from the parametric investigation, for three tube diameters of $50 \mathrm{~mm}$, $75 \mathrm{~mm}$ and $100 \mathrm{~mm}$ and for varying width of the heating element, from $50 \mathrm{~mm}$ up to 90 $\mathrm{mm}$, relating the energy input per heating element and the length of the molten zone created, are summarised in Figure 7. The results indicate a non-linear relationship between length of molten zone and power input, also scaling non-linearly with the diameter of the quartz tube. Such a non-linear relationship is to be expected when the highly non-linear radiative loss terms are accounted for.

The results were normalised to a heat input of $12 \mathrm{~kW} / \mathrm{m}^{2}$, equivalent to a total energy input of approximately $0.094 \mathrm{~kW}$ per heater element. In this way, a correlation 
between the necessary energy input for the onset of melting in the existing equipment, but also in equipment of different geometrical dimensions, with the same proportion of semiconducting material enclosed, can be achieved. For example, from Figure 7, it can be seen that for a zone length of $34 \mathrm{~mm}$ to be achieved in a $50 \mathrm{~mm}$ tube, with a $50 \mathrm{~mm}$ heater element, an energy input of $0.110 \mathrm{~kW}$ per heater, 1.17 times the energy necessary to initiate melting to is required. If the diameter of the tube is doubled, whilst keeping the same width for the heating element, and for the same zone length to be created, then $0.432 \mathrm{~kW}$ per heater, or 3.93 times the energy input for achieving the same length in the $50 \mathrm{~mm}$ tube is required.

If a thin molten zone length is desirable, the results shown in Figure 7 suggest that the region where the gradients are greatest, i.e. large tube diameter and small heater element width, should be selected since any small fluctuation in input energy would result in a small fluctuation of molten zone length.

In order to investigate the influence of the end effects and the inclination of the tube the a further simulation was carried out for the whole tube, with all six heating elements. A single tube diameter of $50 \mathrm{~mm}$ was investigated and the heater elements were set at $18 \mathrm{~kW} / \mathrm{m}^{2}$, resulting in an energy input of $0.14 \mathrm{~kW}$ per heater. The tube was inclined at 6 degrees. Figure 8 presents the temperature distribution along the mid-plane of the tube at mid-height of the charge. Whilst there is a visible end effect due to the convective boundary condition now imposed at each end of the tube, the results are otherwise comparable to those of a simulation for a single element zone. The inclination of the tube has no significant effect upon the temperature distribution within the semiconductor charge. 


\section{Conclusion}

A thermal analysis of the horizontal zone refining of Indium Antimonide has been successfully carried out in the form of a parametric study to investigate the effect of heater and tube dimensions on molten zone length and energy input. The results indicate that more precise control of the molten zone length may be obtained when operating at a larger process diameter, but with narrow heating elements.

The results from the simulation of the whole process confirmed that a modest inclination of the tube does not affect the zone refining process from the heat transfer point of view. Furthermore, the results confirm that modelling a single heating zone is almost identical to when the whole tube, including the six heating elements, is simulated.

These results from this work are expected to be of value in the future optimisation of horizontal zone refining, for both existing equipment and the design of new equipment at larger scales.

\section{Acknowledgements}

The authors are very grateful to P. Burdett of Wafer Technology Ltd for the assistance and support provided during this project.

\section{References}

[1] Pfann, W.G. (1966). Zone Melting, $2^{\text {nd }}$ ed. John Wiley \& Sons, New York.

[2] Spim, J.A., Bernardou, M.J.S. and Garcia, A. (2000). Numerical modelling and optimization of zone refining. Journal of Alloys and Compounds, 298, 299-305. 
[3] Rodway, G.H. and Hunt, J.D. (1989). Optimizing zone refining. Journal of Crystal Growth, 97, 680-688.

[4] Chii-Dong, H., Ho-Ming, Y. and Tzuoo-Lun, Y. (1996). Optimal zone lengths in multi-pass zone-refining processes. Separations Technology, 6, 227-233.

[5] Chii-Dong, H., Ho-Ming, Y. and Tzuoo-Lun, Y. (1999). The optimal variation of zone lengths in multipass zone refining processes. Separation and Purification Technology, 15, 69-78.

[6] Chang, C.E. and Wilcox, W.R. (1975). Heat transfer in vertical zone melting of poor thermal conductors. Journal of Crystal Growth, 28, 288-294.

[7] Kobayashi, N. (1978). Power required to form a floating zone and the zone shape. Journal of Crystal Growth, 43, 417-424.

[8] Wang, J.H. and Kim, D.H. (1997). Numerical analysis of melt/solid interface shape in zone melting recrystallization process. Journal of Crystal Growth, 173, 201299.

[9] Louchev, A.L. (1993). The influence of natural convection on the formation of a molten zone under optical heating. Journal of Crystal Growth, 133, 261-266.

[10] FLUENT Inc. (2001). FLUENT 6.1 User's Guide. FLUENT Europe Ltd, Sheffield Airport Business Park, Europa Link, Sheffield S9 1XU.

[11] Roussopoulos G.S. (2003). Simulation of zone refining for a semiconducting material: Indium Antimonide. MSc Thesis, Cranfield University, Cranfield, UK

[12] Burdett P. (2003). Private Communication 



\begin{tabular}{|l|c|c|}
\hline Melting Point $=809 \mathrm{~K}$ & Solid & Liquid \\
\hline Density $\left(\mathrm{Kg} / \mathrm{m}^{3}\right)$ & 5770 & 7168 \\
\hline Thermal Conductivity $(\mathrm{W} / \mathrm{mK})$ & 4.74 & 12.3 \\
\hline Heat Capacity $(\mathrm{J} / \mathrm{KgK})$ & 209 & 270 \\
\hline
\end{tabular}

Table 1: Thermophysical properties of Indium Antimonide 


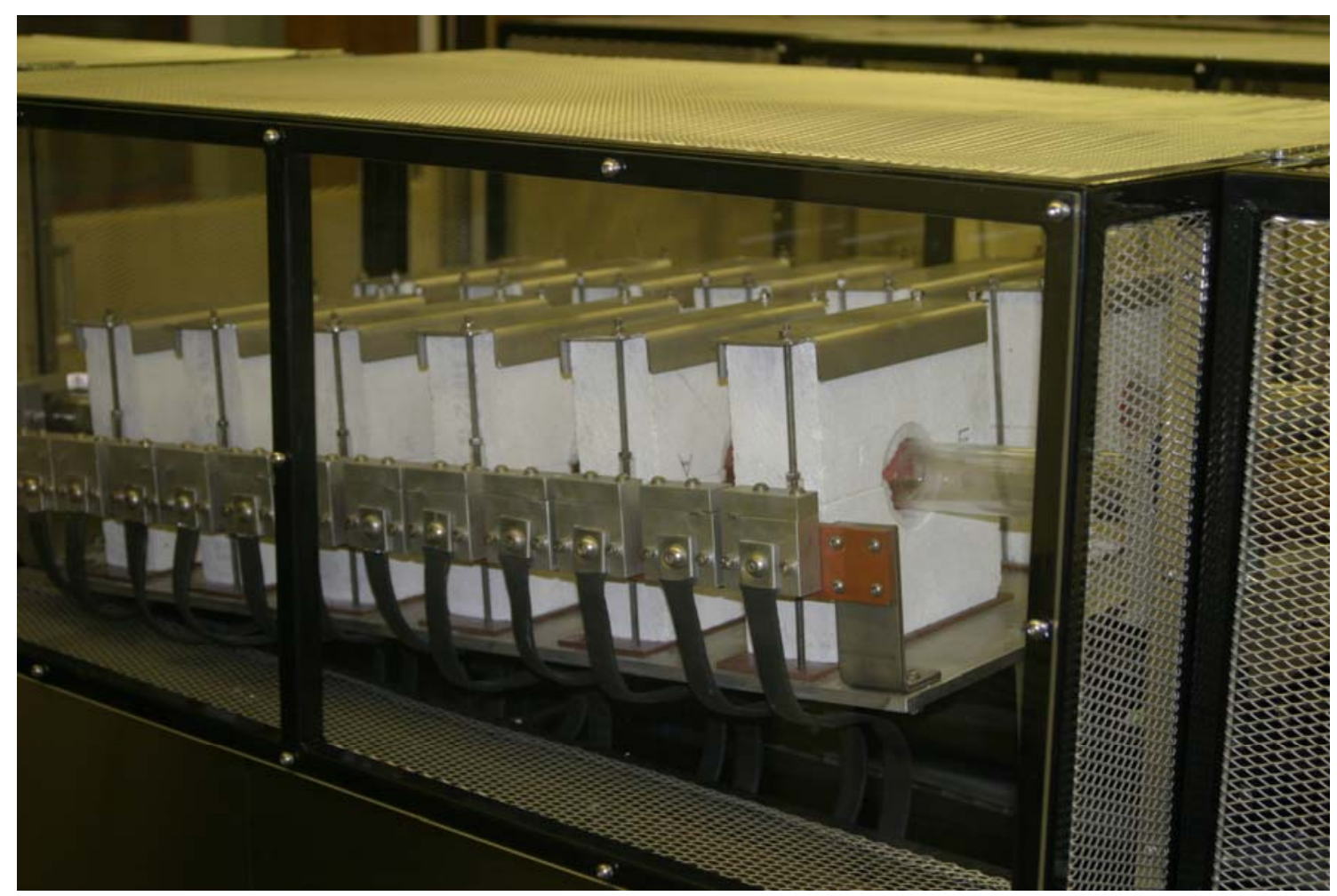

Figure 1: Zone Refining Apparatus 


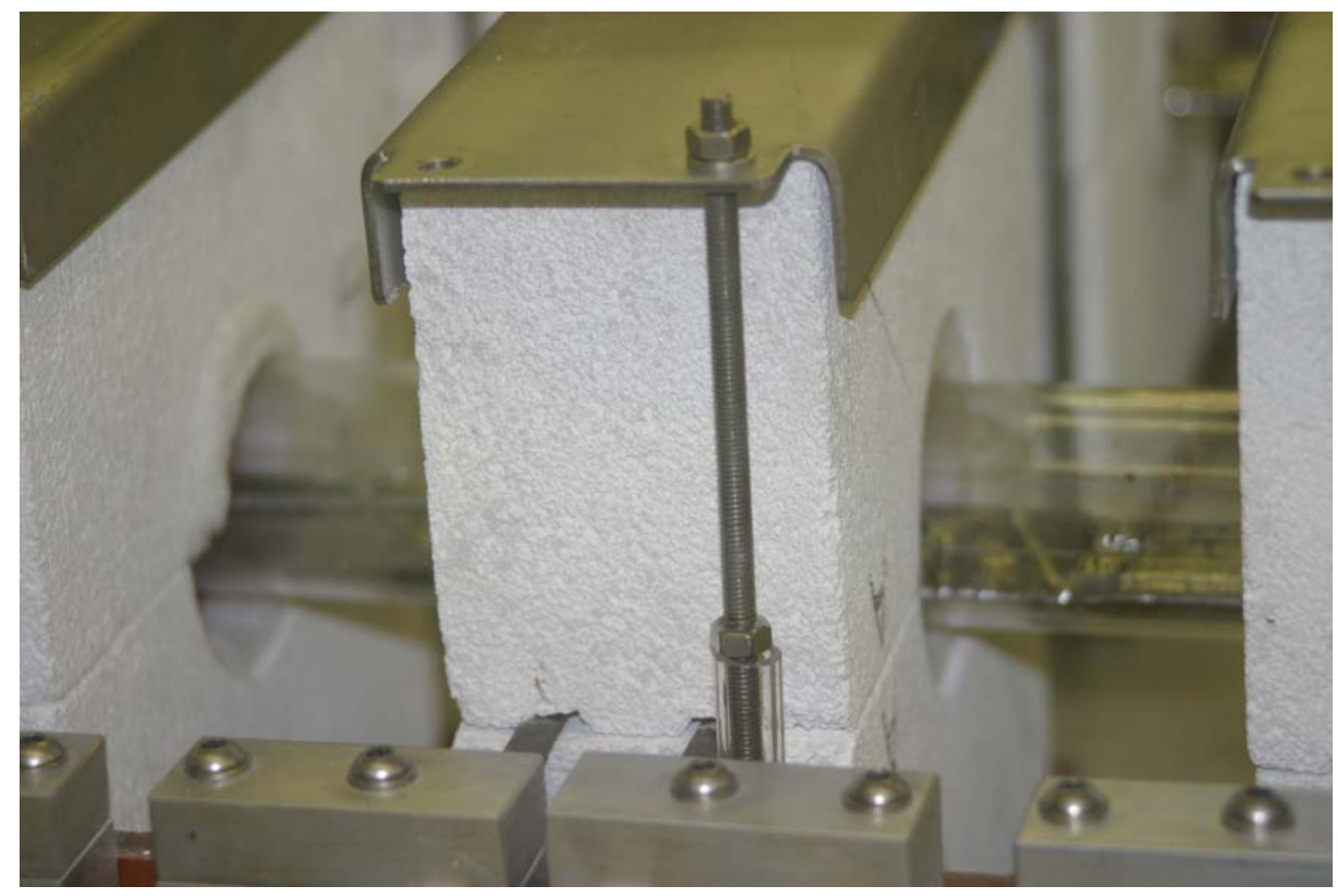

Figure 2: Detailed view of a single heater element 


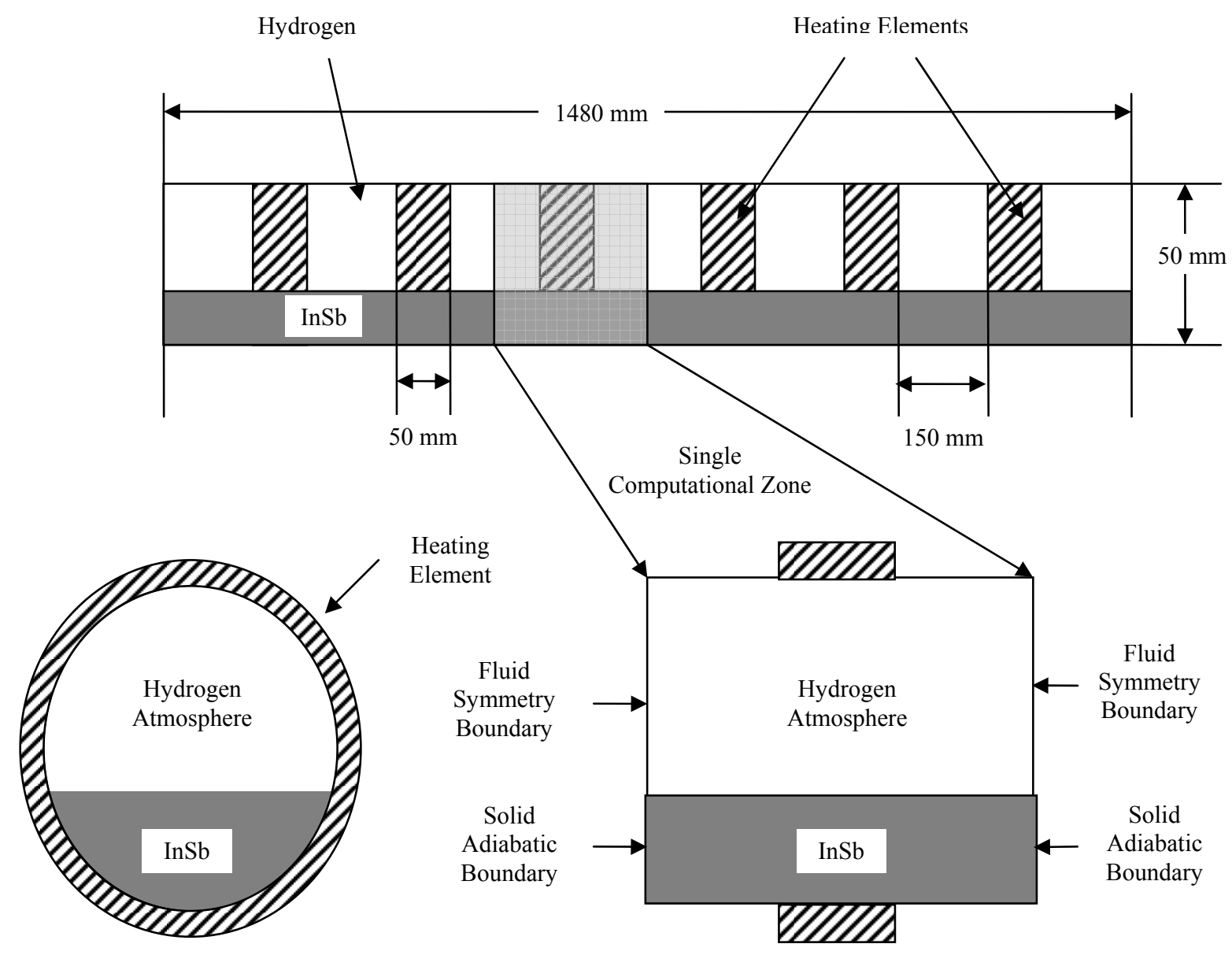

Figure 3: Schematic of the geometry 


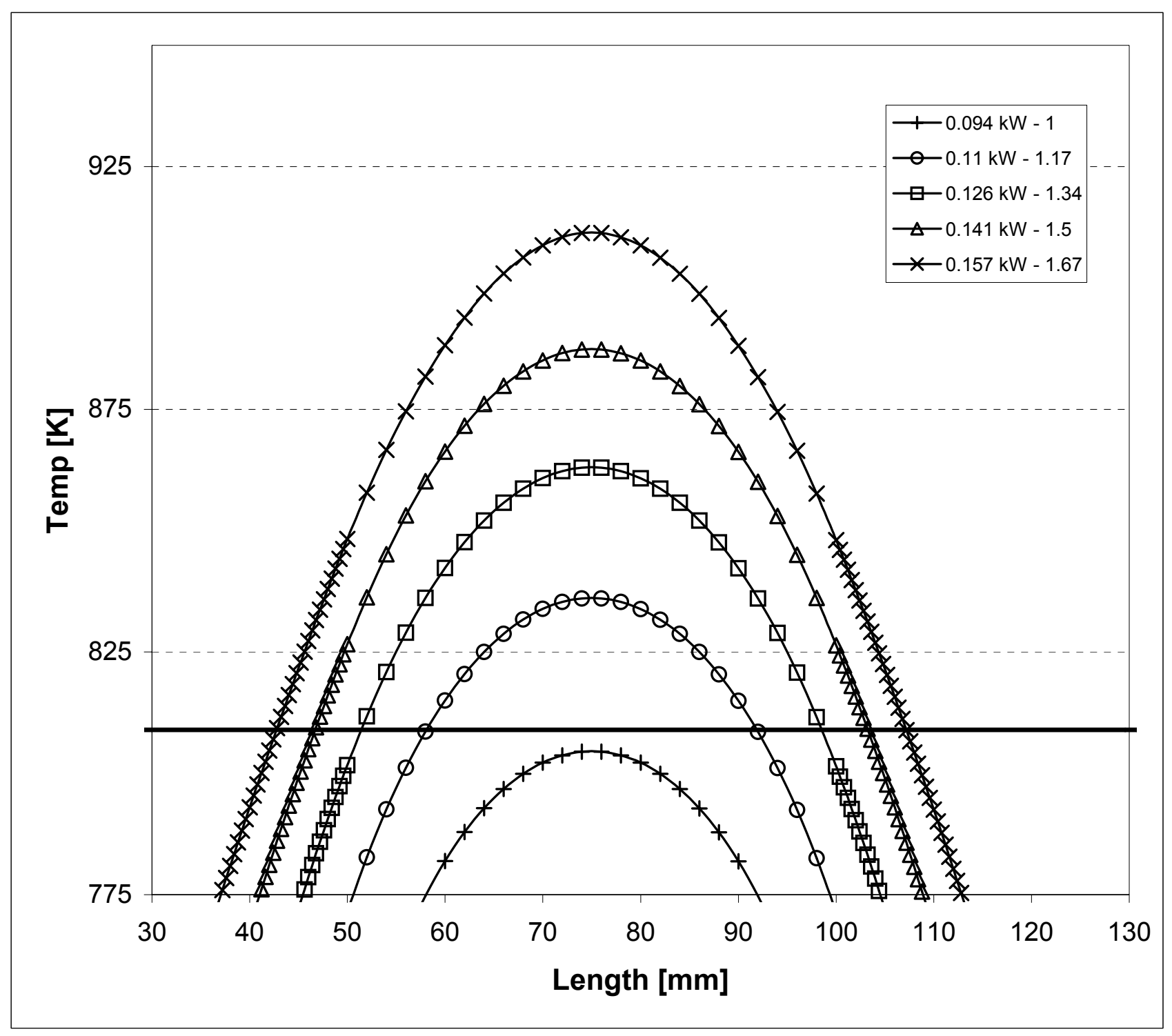

Figure 4: Temperature distribution inside the semiconducting material 


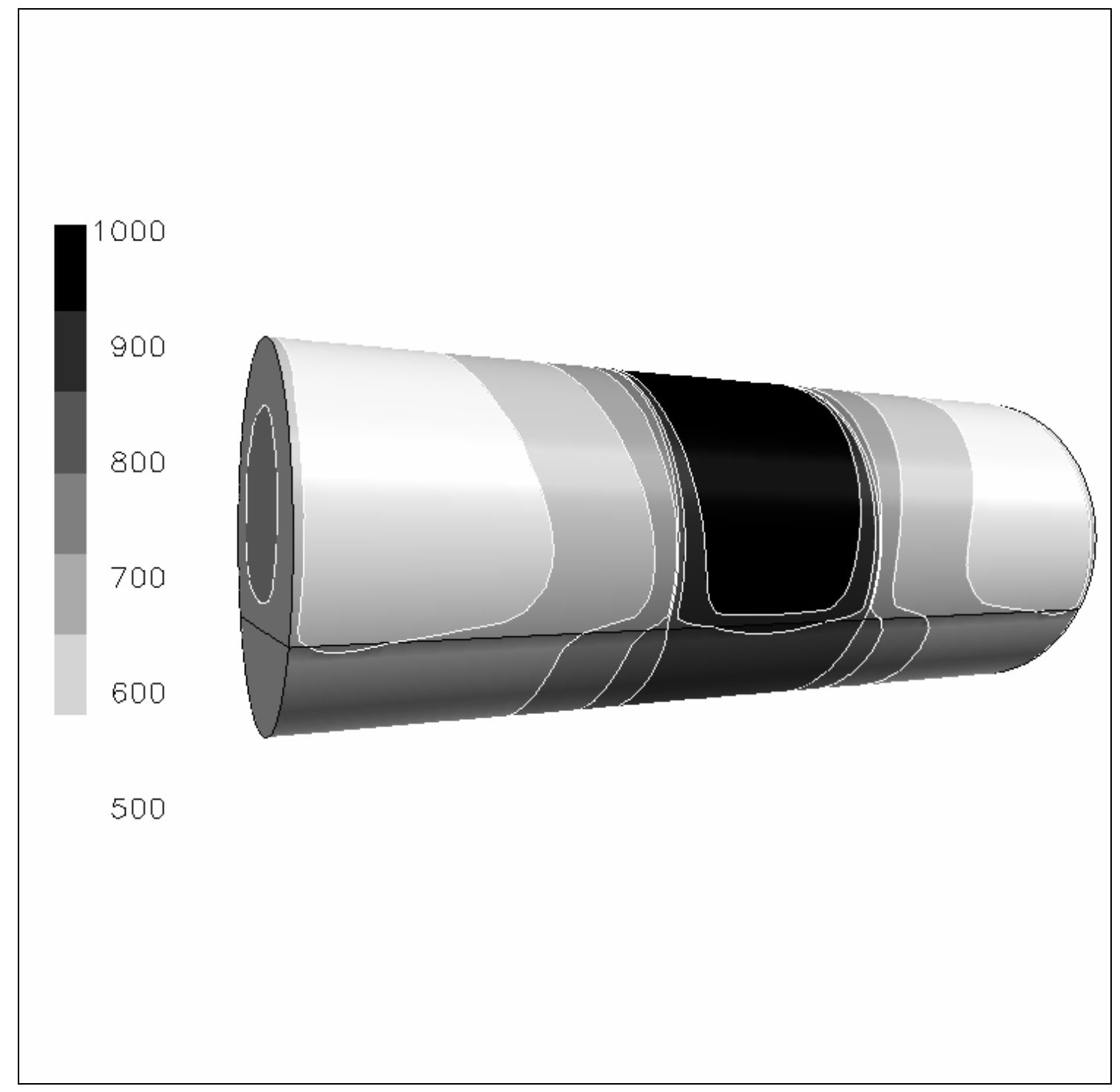

Figure 5a: Surface temperature (K) distribution over the quartz tube 


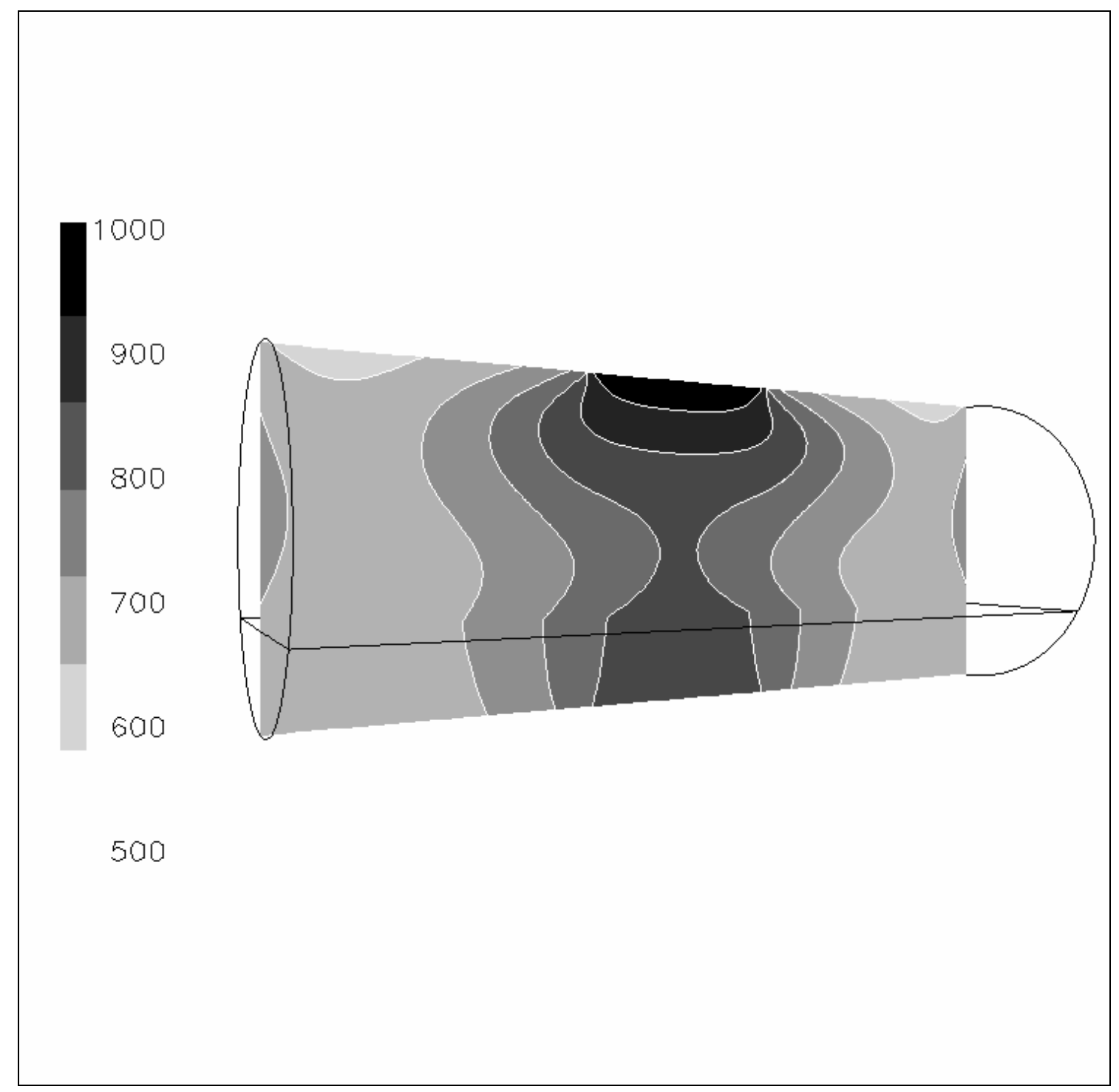

Figure 5b: Contours of temperature $(K)$ on the vertical mid-plane 


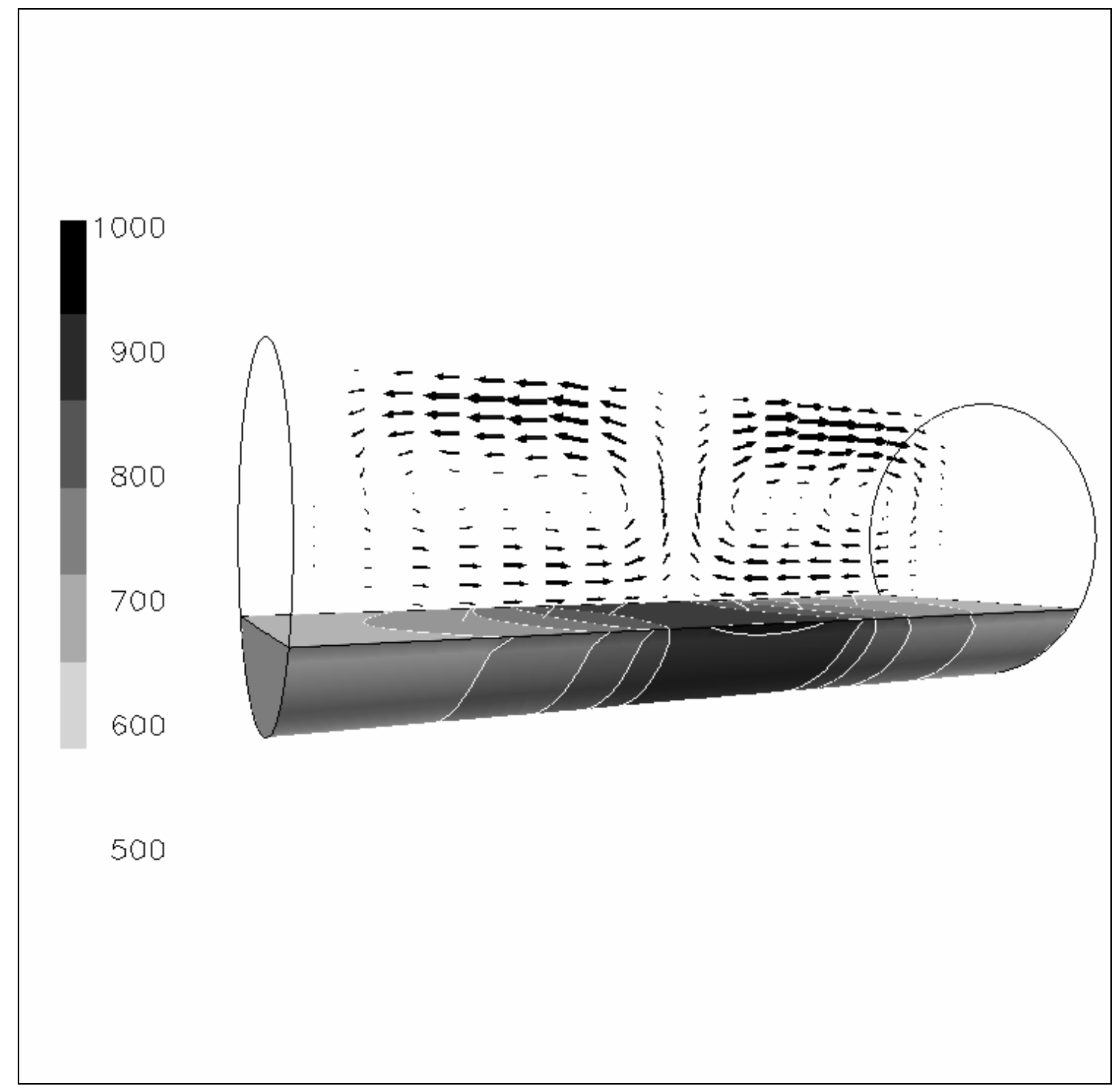

Figure 6: Illustration of the flow field in the hydrogen atmosphere above

the semi-conductor charge

(Velocity vectors scaled by velocity magnitude, charge coloured by temperature $(\mathrm{K}))$ 


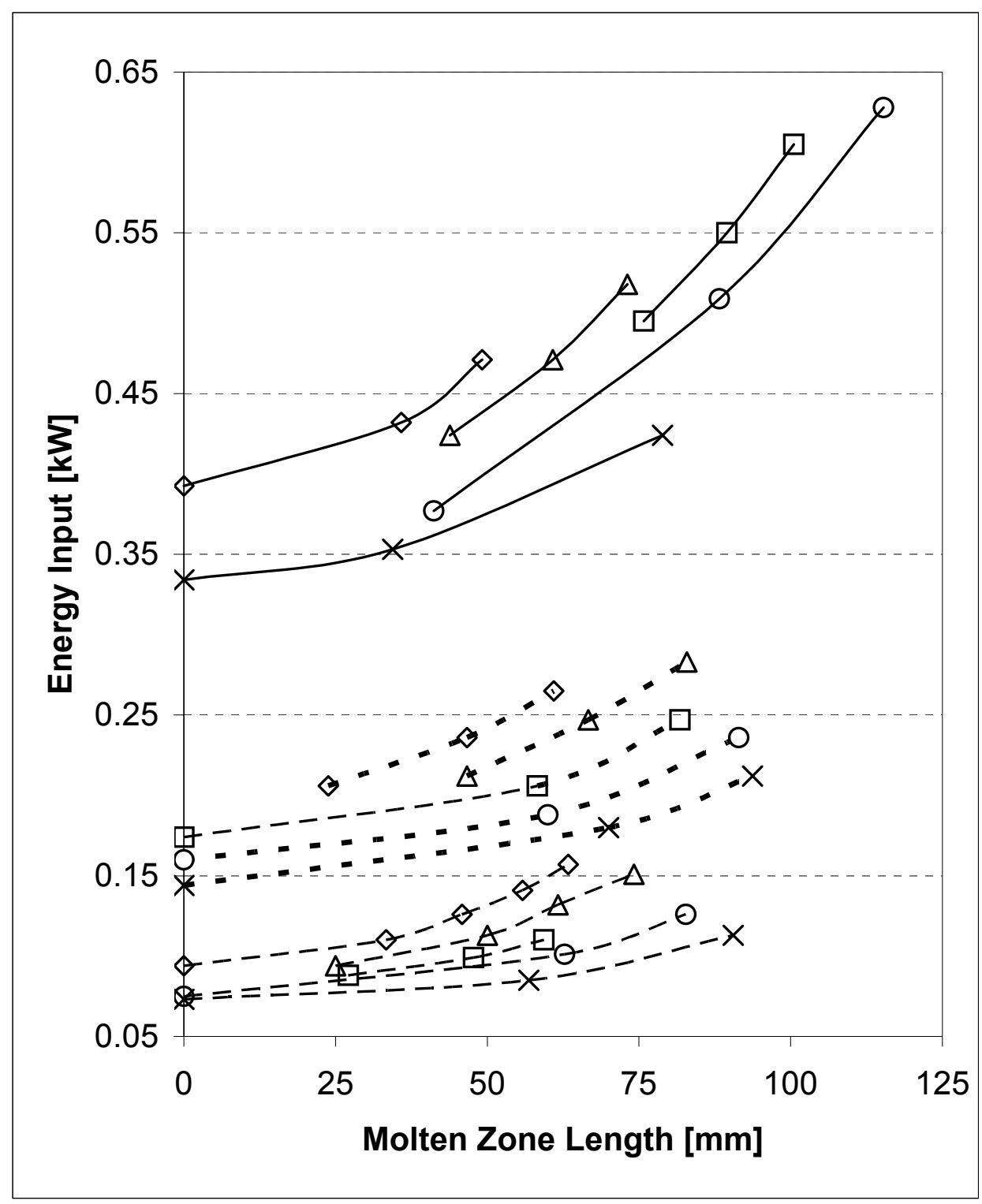

\begin{tabular}{|c|c|}
\hline--- & Diameter of the tube $50 \mathrm{~mm}$ \\
\hline$\cdots \cdot$ & Diameter of the tube $75 \mathrm{~mm}$ \\
\hline- & Diameter of the tube $100 \mathrm{~mm}$ \\
\hline$\triangle$ & Width of the heating element $50 \mathrm{~mm}$ \\
\hline$\triangle$ & Width of the heating element $60 \mathrm{~mm}$ \\
\hline$\square$ & Width of the heating element $70 \mathrm{~mm}$ \\
\hline 0 & Width of the heating element $80 \mathrm{~mm}$ \\
\hline$\times$ & Width of the heating element $90 \mathrm{~mm}$ \\
\hline
\end{tabular}

Figure 7: Energy input and zone length as a function of tube diameter and heater width 


\begin{tabular}{|l|c|}
\hline & Diameter of the tube $50 \mathrm{~mm}$ \\
\hline & Diameter of the tube $75 \mathrm{~mm}$ \\
\hline & Diameter of the tube $100 \mathrm{~mm}$ \\
\hline & Width of the heating element $50 \mathrm{~mm}$ \\
\hline & Width of the heating element $60 \mathrm{~mm}$ \\
\hline & Width of the heating element $70 \mathrm{~mm}$ \\
\hline & Width of the heating element $80 \mathrm{~mm}$ \\
\hline
\end{tabular}




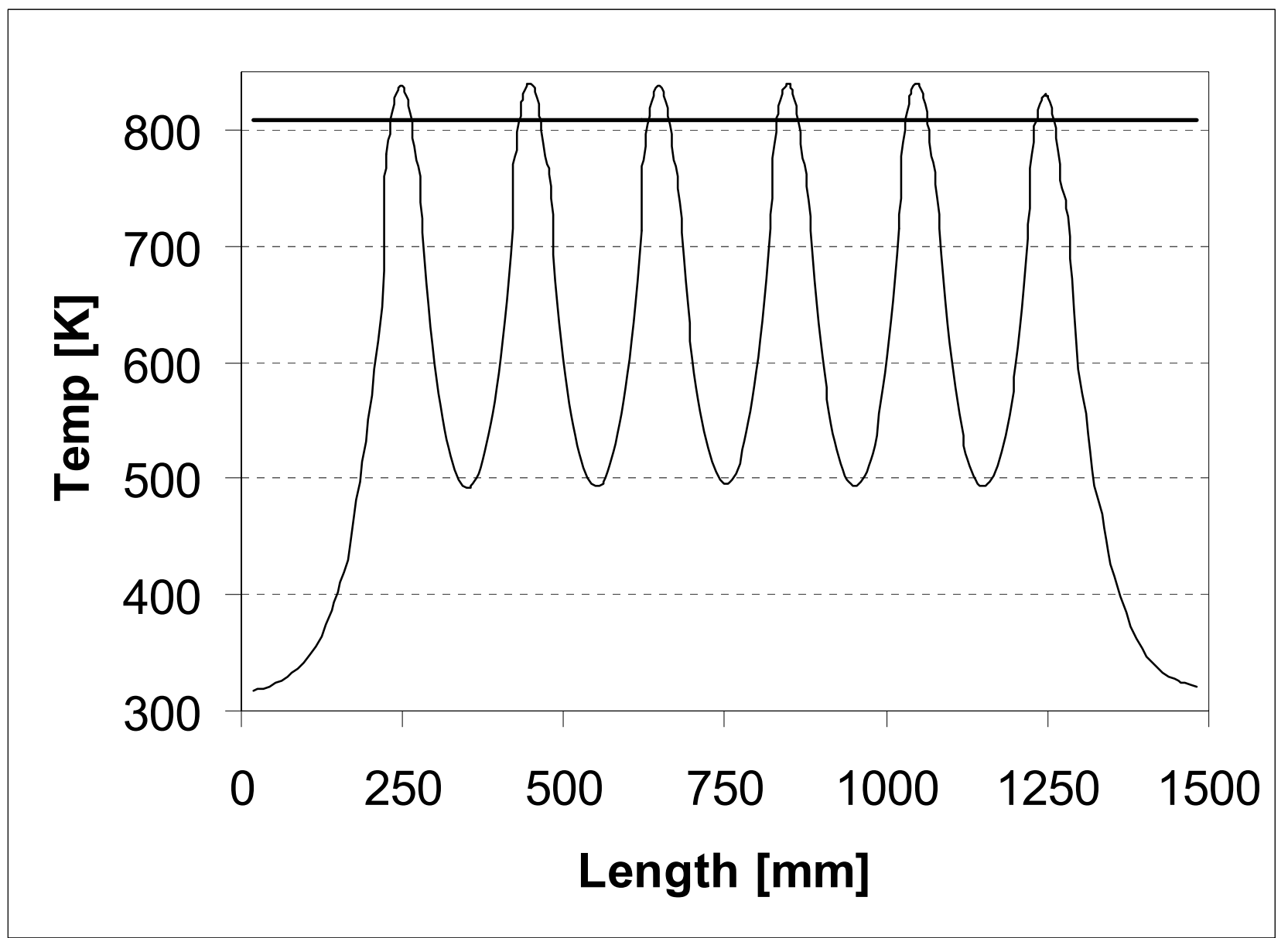

Figure 8: Temperature distribution on the mid-plane at mid-height along the whole tube 